\title{
On Sentence Representations for Propaganda Detection: From Handcrafted Features to Word Embeddings
}

\author{
André Ferreira Cruz and Gil Rocha and Henrique Lopes Cardoso \\ Laboratório de Inteligência Artificial e Ciências dos Computadores (LIACC) \\ Departamento de Engenharia Informática, \\ Faculdade de Engenharia da Universidade do Porto \\ Rua Dr. Roberto Frias, 4200-465 Porto, Portugal \\ \{andre.ferreira.cruz, gil.rocha, hlc\}@fe.up.pt
}

\begin{abstract}
Bias is ubiquitous in most online sources of natural language, from news media to social networks. Given the steady shift in news consumption behavior from traditional outlets to online sources, the automatic detection of propaganda, in which information is shaped to purposefully foster a predetermined agenda, is an increasingly crucial task. To this goal, we explore the task of sentence-level propaganda detection, and experiment with both handcrafted features and learned dense semantic representations. We also experiment with random undersampling of the majority class (nonpropaganda) to curb the influence of class distribution on the system's performance, leading to marked improvements on the minority class (propaganda). Our best performing system uses pre-trained ELMo word embeddings, followed by a bidirectional LSTM and an attention layer. We have submitted a 5-model ensemble of our best performing system to the NLP4IF shared task on sentence-level propaganda detection (team $L I A C C$ ), achieving rank 10 among 25 participants, with 59.5 F1-score.
\end{abstract}

\section{Introduction}

Propaganda shapes information in order to purposefully influence people's mindset and advance a predetermined agenda. The NLP4IF shared task on propaganda detection challenged participants to build systems capable of sentence-level (SLC) or fragment-level (FLC) detection of propagandistic texts (Da San Martino et al., 2019). We have participated on the SLC track, hence this will be the focus of this paper.

The rise of fake (Allcott and Gentzkow, 2017), hyperpartisan (Silverman et al., 2016), and propagandistic news on social media and online news outlets calls for improved automatic detection of bias in texts. However, any and all attempts at automated regulation of online content have freedom of speech implications, and risk unintended cen- sorship (Akdeniz, 2010). Mindful of these considerations, we experiment with a set of handcrafted and interpretable stylometric features, together with a model based on Gradient Boosted Trees (Drucker and Cortes, 1996), thus facilitating inspection of what it is that the model has learned.

In addition, aiming for a better performance to the detriment of the model's interpretability, we experiment with deep neural networks, supplied with word embeddings learned on large external corpora, as this combination is the state-of-theart for several natural language processing (NLP) tasks (Peters et al., 2018; Devlin et al., 2018; Akbik et al., 2019). Nonetheless, some degree of interpretability is maintained through the use of attention layers (Bahdanau et al., 2014), enabling inspection of which time-steps (words) the model is considering when making a prediction.

The provided train dataset consists of 350 articles, with a total of 16,965 sentences $-4,720$ of which are labeled propaganda, and the remaining 12,245 labeled as non-propaganda. This class imbalance leads supervised learning models to favor predicting the majority class (non-propaganda), severely impacting performance on the minority class (Japkowicz and Stephen, 2002). In order to tackle this problem, we train all systems on a balanced version of the provided dataset, by means of random undersampling of the majority class, as this technique has been shown to have good results on several NLP tasks (Japkowicz and Stephen, 2002; Prusa et al., 2015).

The rest of the paper is organized as follows. Section 2 describes data pre-processing and feature selection, and details all tested models and their architectures. Section 3 analyzes our models' performance, analyzes attention-weight plots, and discusses results. Finally, Section 4 draws conclusions and sketches future work. 


\section{System Description}

We propose an approach based on a selection of handcrafted features paired with a Gradient Boosted Trees (GBT) model, as well as an approach based on learned dense semantic representations (word embeddings) paired with different deep-learning models. This Section describes the data pre-processing and feature selection, the choice of word embeddings, and the tested models and their hyperparameters.

\subsection{Data Pre-processing}

We tokenize sentences into words using Spacy (Honnibal and Montani, 2017). We standardize quotation marks (left and right, single and double), as well as single grave and acute accents, as all these characters may be represented by different unicode characters while portraying the same meaning.

\subsection{Feature Selection}

We use a small set of linguistically-inspired style and complexity features, already proven to have good performance on a similar bias-detection task - hyperpartisan news detection (Cruz et al., 2019). Some of the features portray the article in which each sentence is incorporated, while others portray the sentence itself. Our features are as follow:

- num_sentences: total number of sentences in the article;

- avg_sent_char_len: average character-length of article's sentences;

- var_sent_char_len: variance of characterlength of the article's sentences;

- actual_sent_char_len: character-length of current sentence;

- avg_word_len: average of character-length of this sentence's words;

- var_word_len: variance of character-length of this sentence's words;

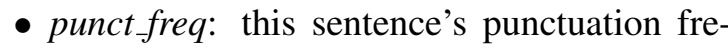
quency;

- capitalffreq: this sentence's capital-case frequency;
- type-token-ratio over lemmatized words a measure of vocabulary diversity and richness (Johnson, 1944).

- TF-IDF (Robertson, 2004) vector for the 50 most frequent unigrams and bi-grams, whose document frequency does not exceed $95 \%$.

\subsection{Contextualized Word Representations}

Deep-learning models proposed in this paper are supplied with dense word representations, generated from the pre-trained ELMo model (Peters et al., 2018). We use the Flair library (Akbik et al., 2019) to generate contextualized 3072-dimensional representations for each input word (concatenation of outputs from three 1024dimensional layers). These embeddings are a function not only of the word itself but also of its context, enabling word disambiguation into different semantic representations.

We crop sentences to a maximum of 50 words, as a compromise between the representation's expressiveness and its computational cost (affecting only $3.7 \%$ of longer samples, see Figure 1). Shorter sentences are padded out with zeros.

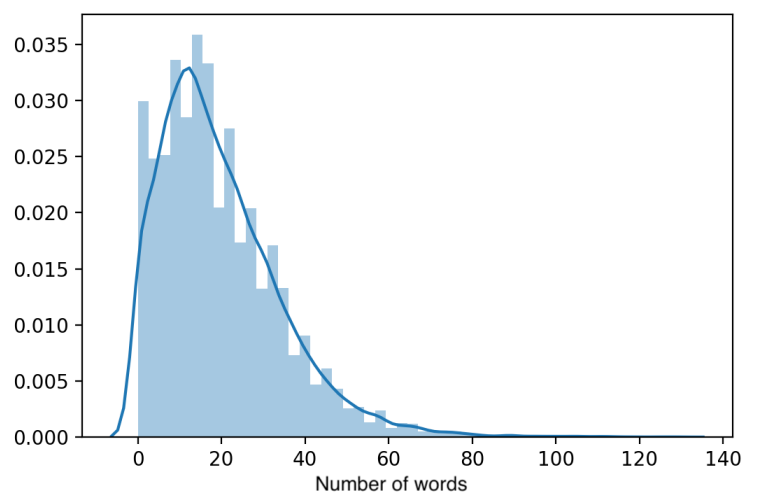

Figure 1: Distribution of sentence length.

\subsection{Models \& Architectures}

As mentioned, we pair the data from handcrafted features with a Gradient Boosted Trees (GBT) model (Drucker and Cortes, 1996). Table 1 shows all hyperparameter values set for the GBT model. These values are the result of extensive grid searching, optimizing for F1-score (the task's official metric), and selecting the best performing model on 5-fold cross-validated results.

Additionally, we devise two deep-learning models to pair with word embedding representations. 


\begin{tabular}{|c|c|}
\hline estimators & 100 \\
\hline learning-rate & 0.1 \\
\hline loss & exponential \\
\hline max. tree depth & 10 \\
\hline min. samples at leaf & 10 \\
\hline min. samples to split & 2 \\
\hline
\end{tabular}

Table 1: Hyperparameter values for GBT.

The BiLSTM model consists of a bidirectional long short-term memory layer (Gers et al., 2000). The last hidden time-step, concatenated from both directions, is then passed through a final fully connected layer followed by a sigmoid activation function. The $A B L$ (Attention-based Bidirectional LSTM) model is similar to the BiLSTM model, with an added attention layer (Bahdanau et al., 2014) operating over the hidden LSTM representations. Figure 2 shows this model's architecture. We use $40 \%$ dropout (Srivastava et al., 2014) on the initial embeddings, and 20\% dropout on all remaining hidden-layers. All LSTM layers use 50 as the number of features of the hidden state.

For training, we use the Adam optimizer (Kingma and $\mathrm{Ba}, 2014$ ) with default parameters, and Binary Cross-Entropy as the loss function. The batch size was set to 16 , and training was stopped after 25 epochs, with early stopping upon 5 consecutive non-improving epochs on validation loss.

Deep-learning models were implemented using PyTorch (Paszke et al., 2017), and GBT using scikit-learn (Pedregosa et al., 2011).

\section{Results and Discussion}

Table 2 shows the results of all models over 5fold cross-validation on the provided SLC training data. The top rows correspond to systems trained on a balanced version of the provided dataset, by means of random undersampling of the majority class (Japkowicz and Stephen, 2002), as an attempt to tackle the class imbalance on the original dataset (only $27.8 \%$ of which corresponds to propaganda sentences).

On the balanced dataset, the $A B L$ model is the best-performing on both F1-score (official task metric) and accuracy, while BiLSTM achieved the best F1-score on the original data. $G B T$ has a surprisingly inferior F1-score on the original data (32.6 points vs 53.0 points on the F1-metric for BiLSTM), but suffers the largest boost when com-

\begin{tabular}{l|cccc} 
Model & $\boldsymbol{F 1}$ & $P$ & $R$ & $A$ \\
\hline ABL Balanced & 75.0 & 71.9 & 78.5 & 73.9 \\
BiLSTM Balanced & 74.7 & 69.5 & 80.7 & 72.6 \\
GBT Balanced & 67.7 & 65.8 & 69.6 & 66.7 \\
\hline BiLSTM & 53.0 & 60.7 & 48.3 & 76.5 \\
ABL & 52.1 & 62.6 & 46.0 & 77.0 \\
GBT & 32.6 & 38.0 & 28.7 & 67.1 \\
\hline
\end{tabular}

Table 2: Propaganda detection performance over 5-fold cross-validation. Models are ordered by decreasing F1score (the task's official metric).

\begin{tabular}{l|ccc} 
Model & $\boldsymbol{F 1}$ & $P$ & $R$ \\
\hline Best (team ltuorp) & 63.2 & 60.3 & 66.5 \\
Ours (ABL-Balanced-Ens) & 59.5 & 50.9 & 71.6 \\
\hline
\end{tabular}

Table 3: Official results for propaganda detection task (on withheld test data).

pared with its training on the balanced data (67.7 F1-score). Nonetheless, models based on word embeddings (BiLSTM \& $A B L$ ) perform far better than those based on a handcrafted selection of features $(G B T)$. This is expected, as $n$-grams fail to encode the text as a sequence, and fail to carry the meaning and relations between each word, which are known to be encoded in word embeddings (Peters et al., 2018).

Regarding the effectiveness of training on a balanced dataset, all systems saw dramatically increased performance on metrics relative to the positive class (labeled propaganda), accompanied by small decreases of overall accuracy. This is expected, as we are effectively depriving the model of useful samples from the majority class (labeled non-propaganda), but remarkably beneficial as can be seen by the improved F1-scores.

Our submission to the task was a 5-member $A B L$ ensemble (ABL-Balanced-Ens), from 5 cross-validation iterations, trained on the balanced data. This system's predictions were the average of each model's independent prediction. This follows numerous works demonstrating consistent performance improvements when using ensembles of deep-learning classifiers (Peters et al., 2018).

Table 3 presents ours results on the official test data. Our system achieved $59.5 \mathrm{~F} 1$-score, ranking $10^{\text {th }}$ among 25 participants, but lagging only 3.7 F1 points behind the best-performing system. 


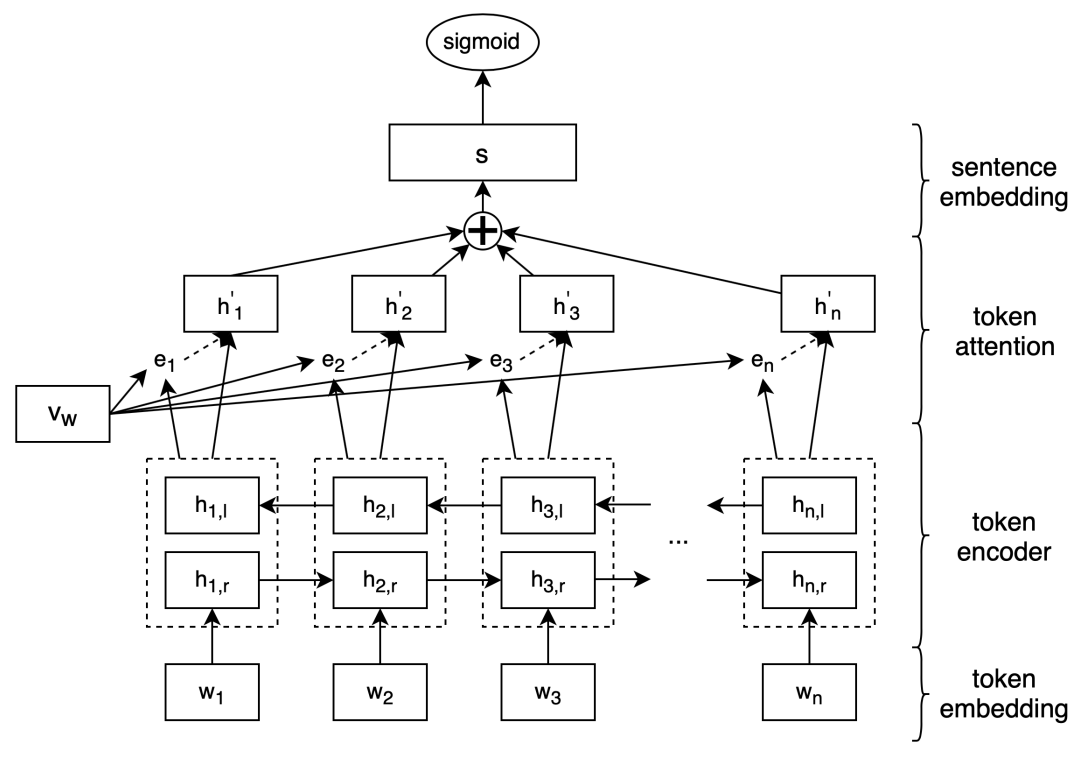

Figure 2: Visualization of ABL (bidirectional LSTM with attention).

\subsection{Analyzing Attention Weights}

Although the predictions of deep learning models are notoriously opaque, the attention layer present in $A B L$ renders some degree of interpretability possible. By analyzing the attention energy associated with each word, we can intuitively extract conclusions regarding which parts of a sentence a model is taking into consideration.

Figure 3 shows a plot of attention energies over a sample article. The model seems to track writing style mostly through verb conjugations (e.g. 'needs', 'given', 'unprecedented'), as well as words with strong connotation which often portray the writer's opinion (e.g. 'wretched deals', 'machination', 'horrify').

From the sentences shown in Figure 3, the model incorrectly classifies the $4^{\text {th }}$ and $5^{\text {th }}$ sentences as non-propaganda (marked $\bullet$ ), although with markedly low confidence ( $8 \%$ and $18 \%$ respectively). All remaining sentences are correctly classified. Through inspection of several attention-plots, intuitively, the model seems to pay close attention to a single opinion-inducing word when classifying a sentence as propaganda, while featuring a broader spread of attention weights when classifying a sentence as non-propaganda. The latter happens for both the $4^{t h}$ and $5^{t h}$ sentences.
He needs medical attention, the kind of treatment you get - only in a hospital.

But it has been made clear to him that if he attempts to go

- to a hospital he will not be given free passage and he will be arrested.

His treatment amounts to the most unprecedented + persecution.

Julian could leave the embassy if his own government, the - government of his homeland, Australia, applied legitimate diplomatic pressure on behalf of its citizen.

- We must ask ourselves why this hasn' t happened.

But that might be one of the so-called "wretched deals"

+ that are being offered Assange.

Some very strange things are being said by senior

- members of these two governments.

The new foreign secretary of the United Kingdom, Jeremy

Hunt, said sarcastically that the British police would offer

+Julian "a warm welcome" when he came out, when he would face serious charges.

Was Hunt referring to a deal which has already been done

- with the United States on extradition?

But this is the milieu of machination around someone who

${ }^{+}$has the right of natural justice concerning his freedom.

Putting aside freedom of speech, the persecution of

+ this man has been something that should horrify all free-thinking people.

Figure 3: Plots of attention-weights. Sentences are marked with + if predicted to be propaganda, and - otherwise. Symbol is colored red if prediction is wrong.

\section{Conclusions and Future Work}

We experimented with several models for sentence-level propaganda detection, exploring both handcrafted features and word embeddings. As expected, deep learning models improve performance to the detriment of feature inter- 
pretability. The best performing model is based on a bidirectional LSTM followed by an attention layer. We have submitted a 5-member ensemble of this model to the NLP4IF shared task, achieving 59.5 F1-score on the official test data, and ranking $10^{\text {th }}$ among 25 participants.

Additionally, we have experimented with random undersampling to tackle the class imbalance on the provided training data. This lead to dramatic performance improvements on all models for metrics related to the minority class, accompanied by a small decrease in accuracy.

For future work, we intend to explore the integration of handcrafted features with word embeddings, to improve both model performance and transparency. We also intend to experiment with ensembles of independent classifiers, from independent feature-sets, in order to capture different facets of this complex problem.

\section{Acknowledgments}

André Ferreira Cruz is supported by the Calouste Gulbenkian Foundation, under grant number 226338. Gil Rocha is supported by a $\mathrm{PhD}$ studentship (with reference SFRH/BD/140125/2018) from Fundação para a Ciência e a Tecnologia (FCT). This research is partially supported by project DARGMINTS (POCI/01/0145/FEDER/031460), funded by FCT.

\section{References}

Alan Akbik, Tanja Bergmann, and Roland Vollgraf. 2019. Pooled contextualized embeddings for named entity recognition. In NAACL 2019, 2019 Annual Conference of the North American Chapter of the Association for Computational Linguistics, pages 724-728.

Yaman Akdeniz. 2010. To block or not to block: European approaches to content regulation, and implications for freedom of expression. Computer Law \& Security Review, 26(3):260-272.

Hunt Allcott and Matthew Gentzkow. 2017. Social media and fake news in the 2016 election. Journal of economic perspectives, 31(2):211-36.

Dzmitry Bahdanau, Kyunghyun Cho, and Yoshua Bengio. 2014. Neural machine translation by jointly learning to align and translate. arXiv preprint arXiv:1409.0473.

André Ferreira Cruz, Gil Rocha, Rui Sousa-Silva, and Henrique Lopes Cardoso. 2019. Team fernandopessa at SemEval-2019 task 4: Back to basics in hyperpartisan news detection. In Proceedings of the 13th International Workshop on Semantic Evaluation, pages 999-1003, Minneapolis, Minnesota, USA. Association for Computational Linguistics.

Giovanni Da San Martino, Seunghak Yu, Alberto Barrón-Cedeño, Rostislav Petrov, and Preslav Nakov. 2019. Fine-grained analysis of propaganda in news articles. In Proceedings of the 2019 Conference on Empirical Methods in Natural Language Processing and 9th International Joint Conference on Natural Language Processing, EMNLP-IJCNLP 2019, EMNLP-IJCNLP 2019, Hong Kong, China.

Jacob Devlin, Ming-Wei Chang, Kenton Lee, and Kristina Toutanova. 2018. Bert: Pre-training of deep bidirectional transformers for language understanding. arXiv preprint arXiv:1810.04805.

Harris Drucker and Corinna Cortes. 1996. Boosting decision trees. In Advances in neural information processing systems, pages 479-485.

Felix A Gers, Jürgen Schmidhuber, and Fred Cummins. 2000. Learning to forget: Continual prediction with 1stm. Neural Computation, 12(10):2451-2471.

Matthew Honnibal and Ines Montani. 2017. spaCy 2: Natural language understanding with Bloom embeddings, convolutional neural networks and incremental parsing. To appear.

Nathalie Japkowicz and Shaju Stephen. 2002. The class imbalance problem: A systematic study. Intelligent data analysis, 6(5):429-449.

Wendell Johnson. 1944. Studies in language behavior: A program of research. Psychological Monographs, 56(2):1-15.

Diederik P Kingma and Jimmy Ba. 2014. Adam: A method for stochastic optimization. arXiv preprint arXiv:1412.6980.

Adam Paszke, Sam Gross, Soumith Chintala, Gregory Chanan, Edward Yang, Zachary DeVito, Zeming Lin, Alban Desmaison, Luca Antiga, and Adam Lerer. 2017. Automatic differentiation in PyTorch. In NIPS Autodiff Workshop.

F. Pedregosa, G. Varoquaux, A. Gramfort, V. Michel, B. Thirion, O. Grisel, M. Blondel, P. Prettenhofer, R. Weiss, V. Dubourg, J. Vanderplas, A. Passos, D. Cournapeau, M. Brucher, M. Perrot, and E. Duchesnay. 2011. Scikit-learn: Machine learning in Python. Journal of Machine Learning Research, 12:2825-2830.

Matthew E. Peters, Mark Neumann, Mohit Iyyer, Matt Gardner, Christopher Clark, Kenton Lee, and Luke Zettlemoyer. 2018. Deep contextualized word representations. In Proc. of NAACL.

Joseph Prusa, Taghi M Khoshgoftaar, David J Dittman, and Amri Napolitano. 2015. Using random undersampling to alleviate class imbalance on tweet sentiment data. In 2015 IEEE international conference 
on information reuse and integration, pages $197-$ 202. IEEE.

Stephen Robertson. 2004. Understanding inverse document frequency: on theoretical arguments for idf. Journal of documentation, 60(5):503-520.

Craig Silverman, Lauren Strapagiel, Hamza Shaban, Ellie Hall, and Jeremy Singer-Vine. 2016. Hyperpartisan facebook pages are publishing false and misleading information at an alarming rate. Buzzfeed News.

Nitish Srivastava, Geoffrey Hinton, Alex Krizhevsky, Ilya Sutskever, and Ruslan Salakhutdinov. 2014. Dropout: a simple way to prevent neural networks from overfitting. The journal of machine learning research, 15(1):1929-1958. 